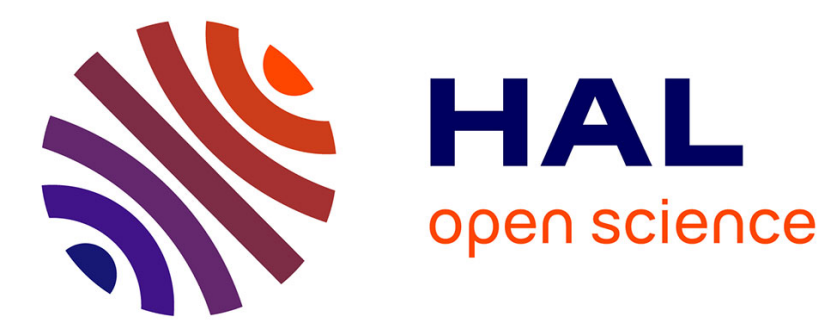

\title{
Optical properties of ordered mesoporous layers of silica
}

Pierre Cheyssac, M. Klotz, E. Søndergård

\section{To cite this version:}

Pierre Cheyssac, M. Klotz, E. Søndergård. Optical properties of ordered mesoporous layers of silica.

Thin Solid Films, 2006, 495, pp.237-242. hal-00459495

\section{HAL Id: hal-00459495 \\ https://hal.science/hal-00459495}

Submitted on 9 Mar 2010

HAL is a multi-disciplinary open access archive for the deposit and dissemination of scientific research documents, whether they are published or not. The documents may come from teaching and research institutions in France or abroad, or from public or private research centers.
L'archive ouverte pluridisciplinaire HAL, est destinée au dépôt et à la diffusion de documents scientifiques de niveau recherche, publiés ou non, émanant des établissements d'enseignement et de recherche français ou étrangers, des laboratoires publics ou privés. 


\title{
Optical properties of ordered mesoporous layers of silica
}

\author{
P. Cheyssac ${ }^{\text {a,* }}$, M. Klotz ${ }^{\text {b }}$, E. Søndergård ${ }^{b}$ \\ ${ }^{a}$ Laboratoire de Physique de la Matière Condensée, Université de Nice, 06108 Nice Cedex 2, France \\ ${ }^{\mathrm{b}}$ Unité Mixte de Recherche CNRS-Saint-Gobain, Surface du Verre et Interfaces, Saint-Gobain, 39 Quai Lucien Lefranc, BP 135, 93303 Aubervilliers Cedex, France
}

Available online 19 October 2005

\begin{abstract}
Mesoporous layers of silica are of interest for many optical applications, including the creation of devices ranging from optical sensors to laser sources with promising characteristics. Their optical characterization goes through the interpretation of measurements. We propose to give experimental results of reflectance and scattering measurements concerning mesoporous layers of silica deposited onto silicium substrates. This work shows that their interpretation in terms of optical indices or dielectric function have to be taken with care since scattering is present, and thereby the Kramers-Kronig relations appear not to be fulfilled.
\end{abstract}

Keywords: Mesoporous materials; Optical properties; Anderson localization

\section{Introduction}

Ordered mesoporous materials differ from bulk matter, exhibiting a highly ordered, monodispersed porosity in the range $2-50 \mathrm{~nm}$, giving rise to a new class of materials. Due to their unique properties, they have received increasing interest from the scientific community since the pioneering work of Beck et al. [1] in 1992. Initially obtained as powders, the synthesis of thin mesoporous films is now achieved [1-4]. These ordered films could be represented as a periodic lattice of holes in an amorphous dielectric matrix, creating an artificial material with a low dielectric constant $[5,6]$ that may be tuned through the amount of pores and their filling [7]. To develop applications [8] from optical sensors [9] to promising laser sources $[10,11]$ as well as three-dimensional optical recording [12], visible light properties need be investigated. It implies the iteration of cycles formulation-optimization-material characterization, a time consuming process. Optical properties are usually described by a set of refractive indices and absorption obtained through reflectance measurements or spectroscopic ellipsometry. An effective dielectric constant may describe the optical properties of a composite medium and may then lead to the filling factor or pores' density $q$ of a mesoporous film. It

\footnotetext{
* Corrresponding author.

E-mail address: pierre.cheyssac@unice.fr (P. Cheyssac).
}

has been recently reported that the angular distribution of light scattered by such a mesoporous film shows an interference pattern [13]. Reflectance usually does not take scattering into account, and so the values of indices deduced from a quick analysis through Fresnel relations have to be taken with care: reflectance does no longer give all optical properties of the sample.

In this paper, we deal with some of the optical characterization of mesoporous materials. We recall the angular distribution of the intensity scattered by an ordered mesoporous silica film. The reflectivity spectra of such film will be presented and applied to the determination of indices of a given sample as long as it retains a physical meaning.

\section{Preparation and structure of the samples}

Each sample consists of a silicon wafer with a thermally oxidized layer, covered by a mesoporous film. Two kinds of samples were realized: $\mathrm{Si}+$ thermal $\mathrm{SiO}_{2}$ (labeled later as $T h$ ), and $\mathrm{Si}+\mathrm{SiO}_{2}+$ a mesoporous layer (Por).

An extended description of the synthesis and ordered structure of mesoporous films have been reported earlier $[14,15]$ and the mechanisms behind their formation are partly understood [16,17]. The films studied in this work were obtained by hydrolysis and condensation of silica alcoxydes in presence of a templating mesophase. More details may be found in Ref. [13]. 

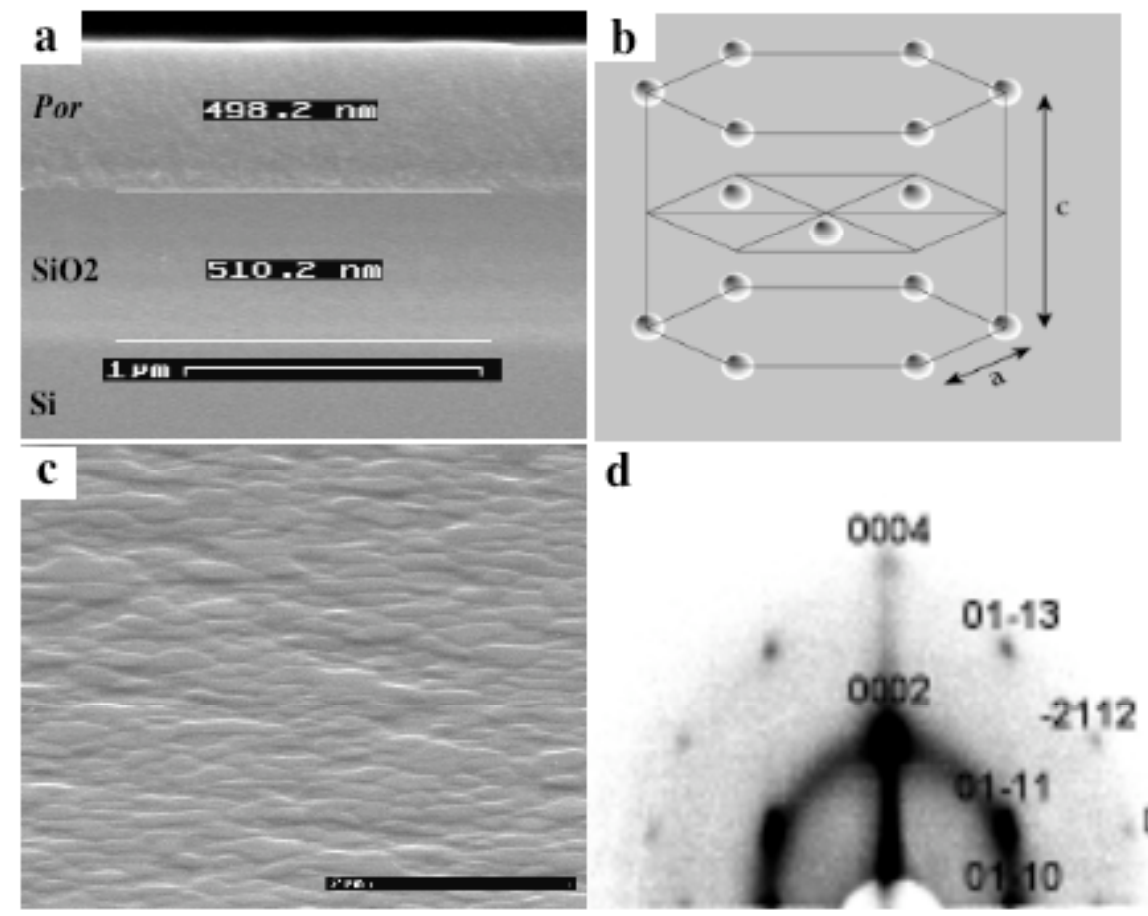

Fig. 1. Structure of the sample a) $\mathrm{SEM}$ view of $\mathrm{Si}+$ thermal $\mathrm{SiO}_{2}$ layer+mesoporous silica film. b) Schematic presentation of the porous film structure. Spheres represent mesopores in $\mathrm{SiO}_{2}$ matrix. c) SEM view of the surface. d) Grazing incidence small angle X-ray diffraction pattern of the mesoporous film. The film is ordered in a $\mathrm{P}_{3} / \mathrm{mmc}$ structure with the $c$-axis perpendicular to the substrate.

A SEM cross-section of the sample is presented in Fig. 1a. The thin film can best be imagined as composed of grains, having all their $c$ axes perpendicular to the substrate Fig. $1 \mathrm{~b}$ but their $a$ axes randomly oriented in the substrate plane [15]. The roughness of the mesoporous surface, shown in the AFM Fig. 1c, is typically $3.8 \mathrm{~nm} \mathrm{r.m.s.}$

The diffraction pattern of the mesoporous film, presented in Fig. 1d, is characteristic of a $\mathrm{P}_{3} / \mathrm{mmc}$ structure; the cell parameters are $a=5.8 \mathrm{~nm}$ and $c=6.9 \mathrm{~nm}$. The ratio $c / a=1.2$ differs from the theoretical value of 1.63 obtained for a close packed hexagonal structure. This is consistent with a $27 \%$ unidirectional shrinkage of the film perpendicular to the substrate $[4,15]$. The mesopores form a unidirectional compressed close packed hexagonal structure. The size of the mesopores was estimated to $3.6 \mathrm{~nm}[18,19]$.

\section{Experimental results}

An original patented installation [20] has been used for the measurements of light scattered by mesoporous films deposited onto $\mathrm{Si}$. The light source is a He-Ne laser tunable at $\lambda_{1}=594$ and $\lambda_{2}=632.8 \mathrm{~nm}$; the beam impinges at normal incidence onto the sample and is focused down to $\sim 100 \mu \mathrm{m}$ diameter while remaining quasi parallel. The Bidirectional Scatter Distribution Function (BSDF) $B S D F=\frac{1}{I_{0} \cos \theta} \frac{\mathrm{d} I}{\mathrm{~d} \Omega}$ is the scattered intensity $\mathrm{d} I$ normalized to the incident intensity $I_{0}$, the solid angle $\mathrm{d} \Omega$ of the receptor and $\cos \theta ; \theta$ being the polar angle. $\log \operatorname{BSDF}\left(f_{\mathrm{x}}\right.$, $f_{\mathrm{y}}$ ), is scaled with grey levels on a 2D diagram, shown in Fig. 2, as a function of the spatial frequencies $f_{\mathrm{x}}, f_{\mathrm{y}}$. The center of the diagram corresponds to the intensity scattered normally to the surface, its borderline is the grazing scattering. When $\lambda$ changes from $\lambda_{1}$ to $\lambda_{2}$, the angular distribution of intensity exhibits well-pronounced minima that remain after an azimuthally averaging of BSDF over $\varphi$; they are given by $\sin$ $\theta_{\min }\left(\lambda_{1}\right)=0.721 \pm 0.02$ and $\sin \theta_{\min }\left(\lambda_{2}\right)=0.582 \pm 0.02$.

Specular reflection spectra $\operatorname{Rexp}(\lambda)$ have been measured from $\lambda=400 \mathrm{~nm}$ up to $800 \mathrm{~nm}$, close to normal incidence with a dedicated apparatus [21]. This lab-built spectrophotometer uses an optical double-path and lock-in amplifier techniques to achieve absolute measurements of reflectance with an accuracy
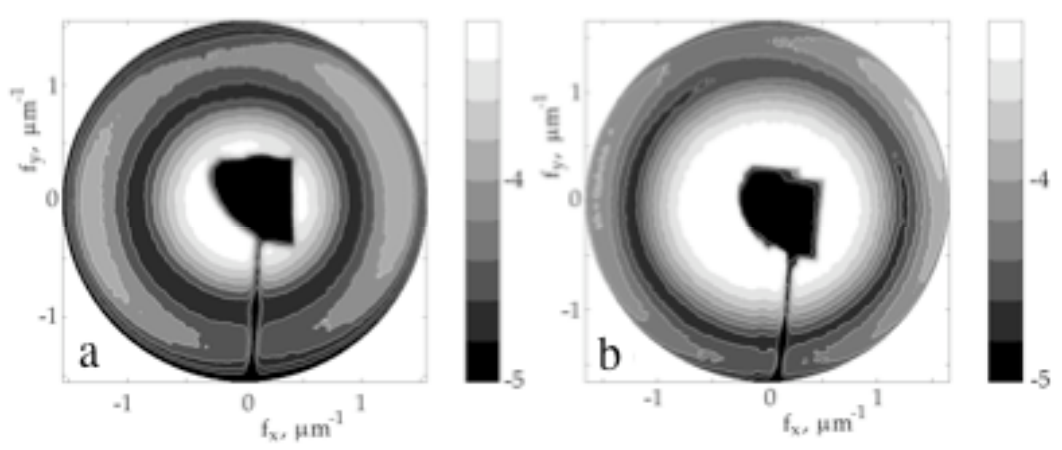

Fig. 2. Mesoporous film, $\log \operatorname{BSDF}\left(f_{\mathrm{x}}, f_{\mathrm{y}}\right)$ as a function of the spatial frequencies: (a) $\lambda=638.8 \mathrm{~nm},(\mathrm{~b}) \lambda=594 \mathrm{~nm}$. 


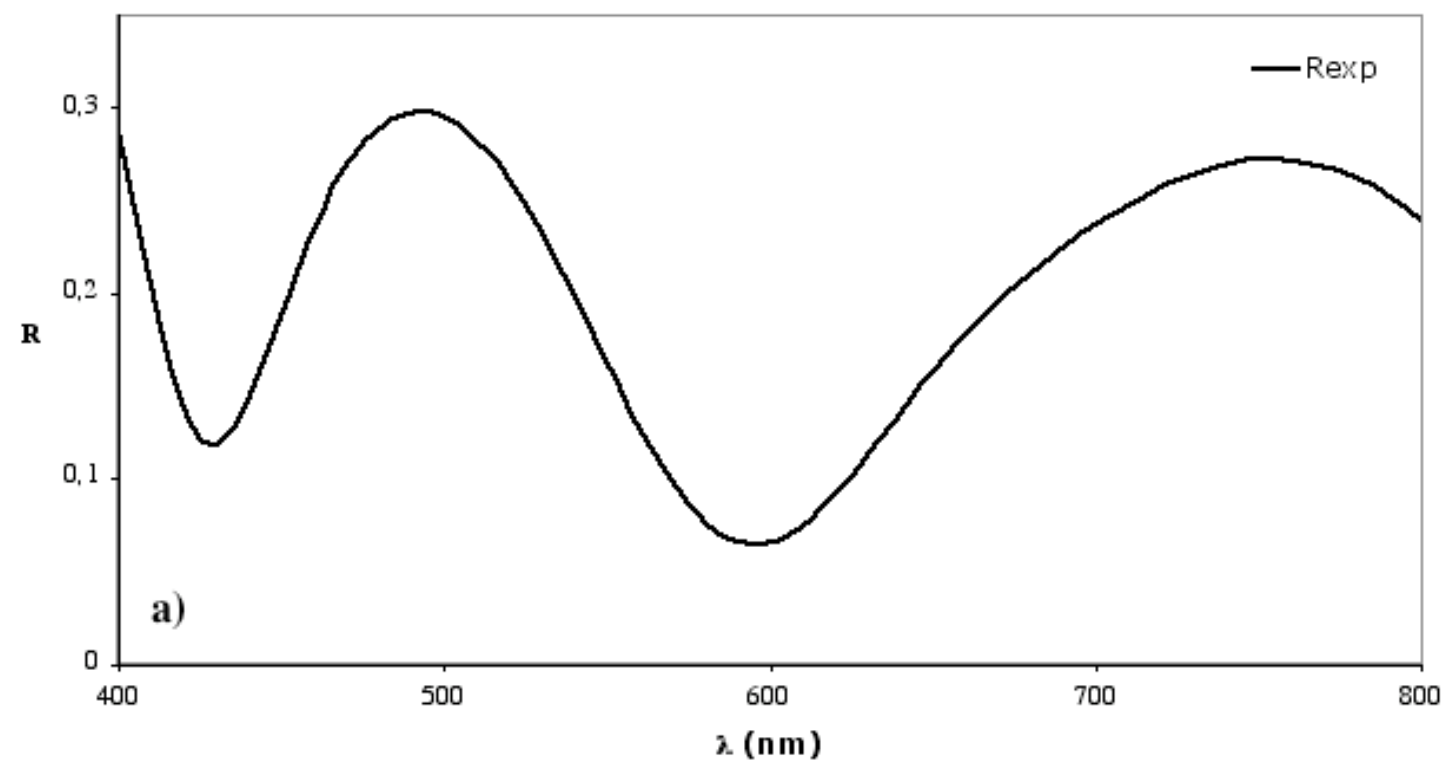

n
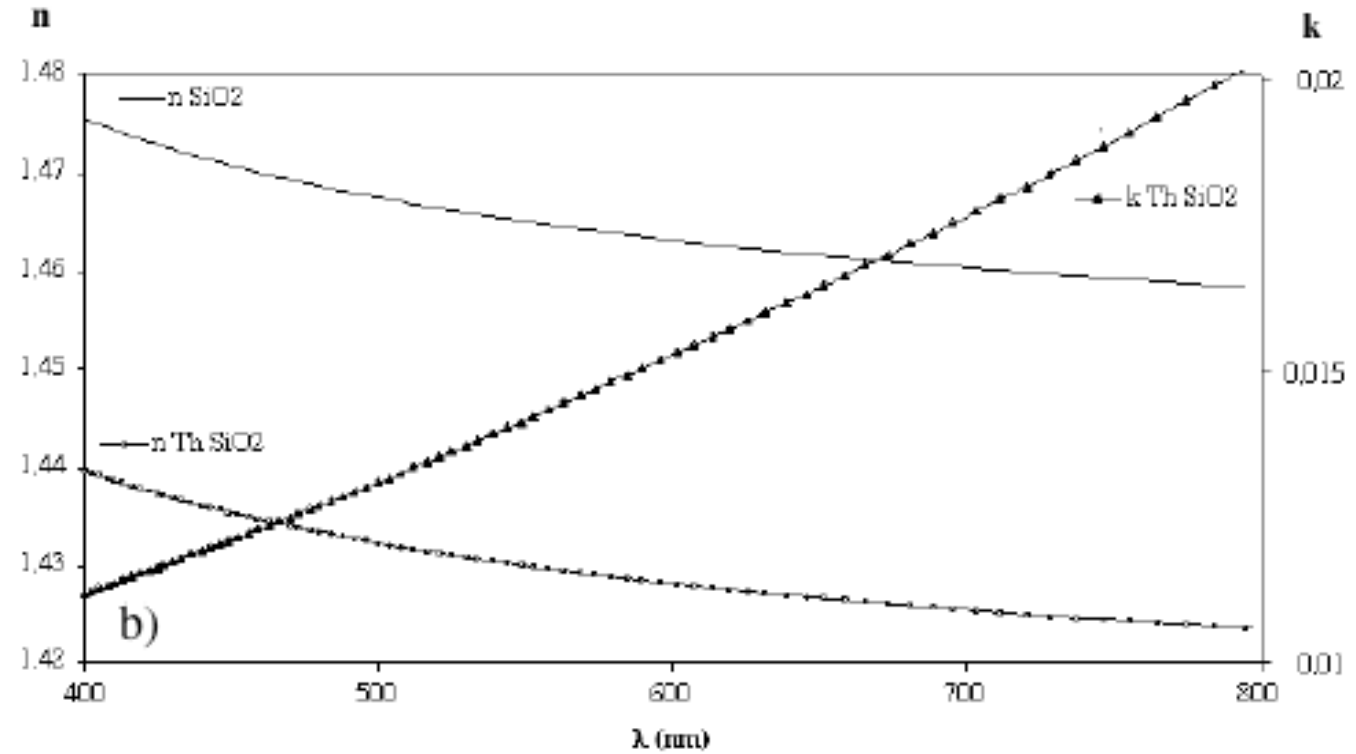

Fig. 3. Reflectance spectrum and corresponding indices of a $T h \mathrm{SiO} 2$ layer deposited onto $\mathrm{Si}$. a) $\operatorname{Rexp}(\lambda)$. b) Calculated indices $n T h=v+i K$ of $\mathrm{SiO}_{2}$; for comparison $\mathrm{n} \mathrm{SiO}_{2}[23]$ is also given.

better than $0.1 \%$. The reflection spectra of $T h$ and Por samples, given in Fig. 3a and 4a, show characteristic interference fringes.

\section{Interpretation}

A plane wave incident normally to the surface of the sample is scattered and exhibits a deep minimum (see Fig. 2a and b) in the direction $\theta_{\min }$ that shifts significantly with $\lambda$. The pores are homogeneously distributed inside the film and exhibit order; each acts as a point source and creates coherent scattering. Assuming that they scatter isotropically, a calculation of the amplitude of all beams scattered in the direction $\theta$ reproduces the behavior [13] of the intensity minimum with $\lambda$.

The interpretation [13] of the reflectance of the $T h$ film has been done as follows. The input are the indices of $\mathrm{Si}$ [22], the output $n T h$ is a Cauchy's-type model [23]. The theoretical reflectance of the multilayer sample is given in the well-known "Principles of optics" textbook by Born and Wolf. The best agreement with Ref. [23] is obtained through a minimization of the difference between experimental and theoretical reflectances. A faint absorption $\kappa T h$ accounts for the scattered energy that may be lost for the reflectance measurements. When $\lambda$ increases, $v T h$ decreases monotonously as it is expected for a transparent medium. The calculated $v T h$, and $\kappa T h$ are given in Fig. $3 \mathrm{~b}$ together with $n T h$ [23].

Thermal $\mathrm{SiO}_{2}$ is known to be highly transparent in the visible. Actually, an imaginary part of the index has been introduced to determine to what extent scattering, which is radiated outside the measured reflected beam, could be represented by an absorbed energy inside the material. Without such correction, the discrepancy between the calculated real part of the index and published values increases.

Hence, as far as scattering is involved, reflectance measurements and the correlated determination of optical indices should be taken with a relative confidence.

Concerning the interpretation of the optical properties of the mesoporous layer, two approaches have been compared. 

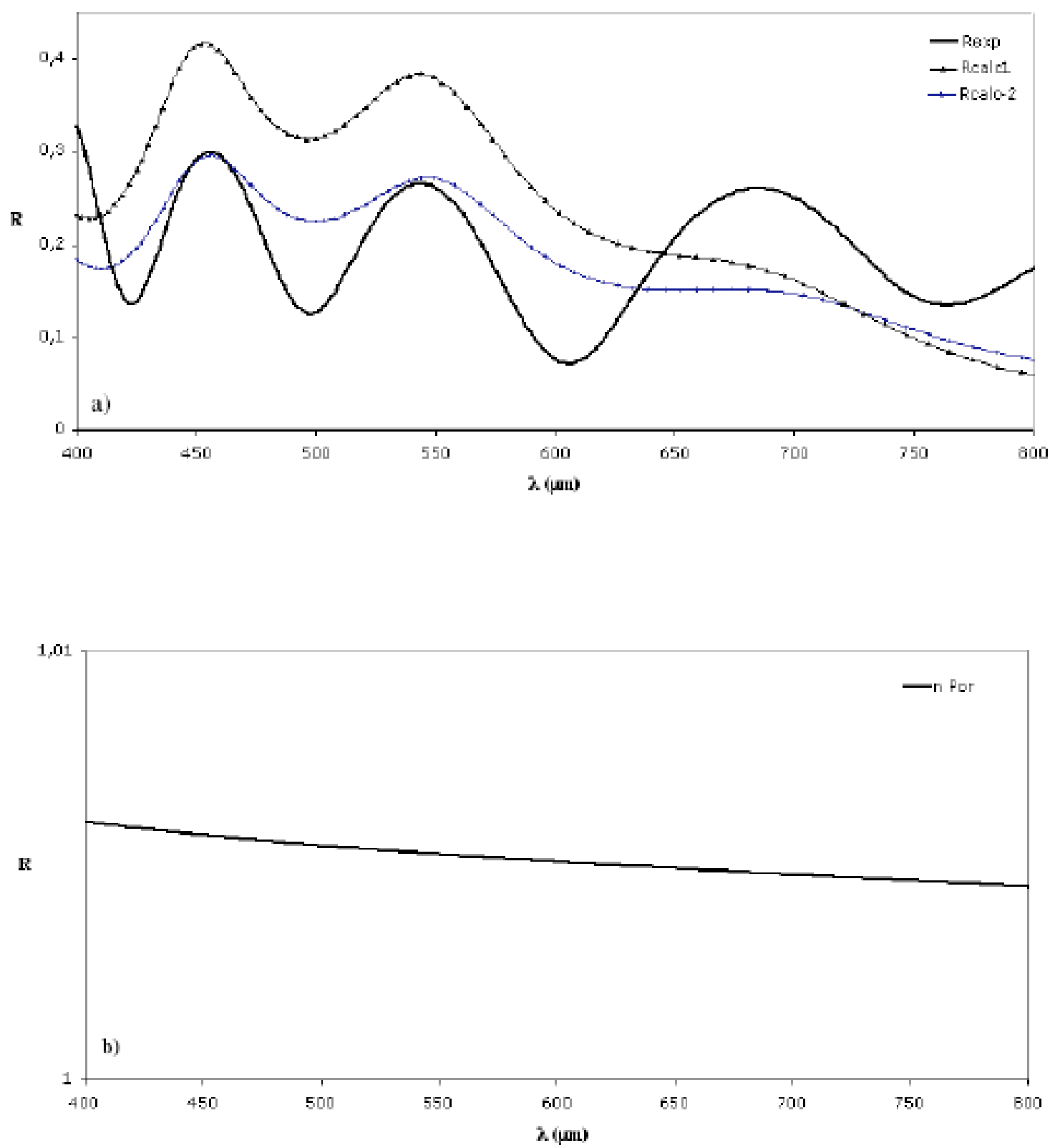

Fig. 4, a) Experimental reflectance $R \exp$ of a mesoporous layer, calculated Fresnel reflectance $R$ calc- 1 and $R$ calc- 2 . b) Corresponding index.

First approach: A theoretical expression of Fresnel reflectance has been written, using the combination of interfaces given by Bom and Wolf and many classical textbooks. SEM gives thicknesses of layers. The chemical composition of the mesoporous layer being almost the same as that of $\mathrm{SiO}_{2}$, the structure makes the difference. A common optical behavior as a function of $\lambda$ may be expected. We assumed that the optical index of the mesoporous layer retains the same Cauchy 's type model [23] as that of $\mathrm{SiO}_{2}$. The reflectance of the mesoporous layer is given in Fig. 4a. Then, $\Delta=R \operatorname{Fresnel}(\lambda)-R \exp (\lambda)$ has been minimized by varying the parameters that enter in the expression of the index nPor. First point of view: the presence of pores, empty or filled with air, characterized by a filling factor $q$ reaching 0.43 , decreases the density of a Por film compared to the bulk silica one's. This should give rise to a lower index of the mesoporous layer compared to that of bulk $\mathrm{SiO}_{2}$. Along these lines, one obtains a calculated Fresnel reflectance $R$ calc-1, shown in Fig. 4a. Silica and pores, that are both transparent in our wavelength range under study, create a two components composite material whose index can be described within the frame of an effective medium theory, although $q$ takes high values. In the calculation, the chosen thickness $d_{0}=497 \mathrm{~nm}$ of the mesoporous layer controls the position of the interference peaks. In both cases, none trial succeeded satisfactorily, but the calculated values of the index shown in Fig. 4b are so close to 1 that it does not make physical sense, reasons of which will be discussed below.

Second point of view: reflectance as described by Fresnel relations has to be modified in order to take into account in one way or another the energy lost by scattering. We have introduced a correction term such as $R$ calc- $2=R$ Fresnel $*\left(\alpha+\beta \lambda^{4}\right)$ presented in Fig. 4a. The position of extrema are identical to those obtained with $R$ calc-1, the index retains quite constant values close to 1 and $R$ calc- 2 is closer to the experimental result than $R$ calc- 1 , although a good agreement cannot be claimed. As far as interferences are present both in reflectance as well as in scattered light, it is very difficult to conclude about the influence of scattering as a decrease or an increase of the reflectance values since multiple interactions exist. 


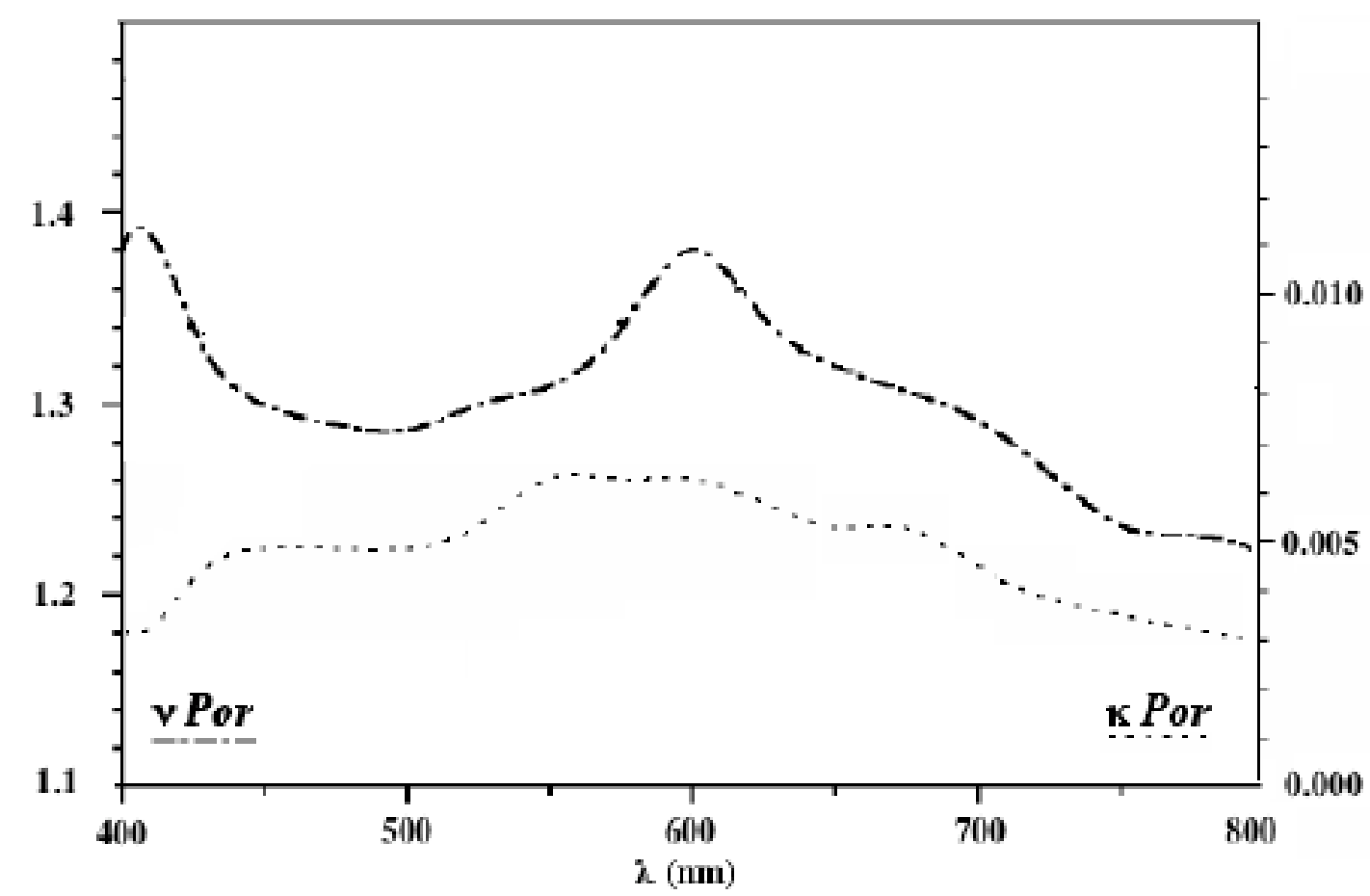

Fig. 5. Calculated indices $n T h=v+i \kappa$ of mesoporous $\mathrm{SiO}_{2}$ obtained from [24].

Second approach: A numerical computation of indices from the reflection spectra has been performed [24], following a method already published [25] and adapted to the determination of indices of thin films deposited onto absorbing substrate. The agreement between $R \exp (\lambda)$ and $R$ calc $(\lambda)$ strongly depends of the range of $\lambda$ under consideration. The as-obtained indices $v T h$ and $\kappa T h$ are given in Fig. 5 ; they seem to show absorption bands. The materials being transparent, the physical meaning of this solution will have to be questioned in the discussion.

\section{Discussion}

The interpretation of the optical properties of the thermal $\mathrm{SiO}_{2}$ layer has already been published [13] but has been recalled here for sake of understanding. For a mesoporous layer, a calculated value of the reflectance has been compared to the experimental one's. Rcalc was given by Fresnel relations, involving indices coming from the literature or an effective medium theory, and also has been modified slightly in a somehow artificial way. Numerical values of indices of a mesoporous layer have been obtained through a minimization procedure of $\Delta=R \operatorname{calc}(\lambda)-R \exp (\lambda)$.

Actually, interpretation of experimental reflectance in terms of optical indices and thicknesses through Fresnel relations means that the film under study satisfies related assumptions, such as homogeneous materials, single or many abrupt interfaces. In our case, mesoporous layers scatter light; then, strictly speaking, specular reflectance does no longer measure the whole energy reflected by the sample, since the scattered energy will not reach the aperture of the detector. Of course, the value of the ratio between the scattered and specular energies will govern the validity of the calculations performed in the frame of the previous assumptions. It is now necessary to question both the obtained values as well as the physical meaning of the approaches.

Let us suppose that scattering is negligible compared to the specular reflection. Then, the calculations performed in the first approach satisfy the previous assumptions. The thickness $d_{0}=$ $497 \mathrm{~nm}$ of the mesoporous layer used in the calculations corresponds very closely to the measured one $490.7 \mathrm{~nm}$. The positions of the extrema are reproduced to a given extent and are controlled by $d_{0}$. Trials to minimize $\Delta$ led to values of $n P o r$ that decreases when $\lambda$ increases, a behavior compatible with that of a transparent medium; and no absorption band appears. Another possibility for a better agreement between $R$ calcl and $R \exp$ would be to introduce a complex valued index with an imaginary part $\kappa$ Por. This hypothesis has been checked: values of $\kappa$ Por that would lead to a better agreement cannot be justified for a transparent medium. $n$ Por retains values $\approx 1$, considerably lower that what could be expected even when one considers that the amount of air in the composite is as high as 0.43 . Effective medium theories, if still applicable but they actually fail for such high $q$, would lead to $1.240<n<1.250$; moreover, the calculation even don't give the number of reflectance extrema.

In our case, scattering is no longer negligible, then one cannot expect finding representative values of index, even an effective one, when inverting Fresnel reflectance because the lost energy is not taken into account in the relations. In order to check if scattering could be the origin of the failure of our procedure, we tried to compensate it in the simplest way: $\lambda$ is $\gg$ diameter of pores, then Mie scattering takes place and radiated energy varies as $\lambda^{-4}$. The simulation of the ad hoc expression $R$ calc- 2 has shown that extrema remain at the same place, but the agreement between $R$ calc- 2 and Rexp increases; however, the index remains at the same low level. Thickness and indices being the only parameters that can be varied in the calculations, the origin of these extended discrepancies has to be found elsewhere.

The second approach [24] has been based upon a numerical resolution of equations established in the literature [25]. It turns out that the set of values that may realize a fit between $R \operatorname{calc}(\lambda)$ and $R \exp (\lambda)$ cannot be represented by a series development of a Cauchy's type model of dielectric. The index takes complex values: its real part exhibits high values and is not monotonous 
as it is expected from effective medium theories applied to a mixture of transparent media such as silica and air or vacuum whatever the filling factor $q$ would be. The imaginary part, which is related to absorption, shows also a non-monotonous variation. Although $n(\omega)$ is not directly related to causality whereas the dielectric function $\epsilon(\omega)$ is, the real and imaginary parts of both $\epsilon$ and $n$ must satisfy a strong requirement for a transparent medium: namely the Kramers-Kronig relations [26]. Hence, they must exhibit the structure correlations asked for by KKR. Fig. 5 does not show such structure correlations, then the as obtained numerical values of the index may solve a set of equations but it appears difficult to attach a physical meaning to these values and their wavelength behavior.

As it has been demonstrated, a mesoporous layer is a strongly scattering medium. Fresnel relations are based on a wave transport in homogeneous medium, abrupt interfaces well localized in space that create reflection, and no interaction between interfaces is considered. On the contrary, the mesopores constitute a distribution of interfaces not localized but spread in the whole material, each pore being in the near field radiated by its neighbour so interactions are present everywhere inside. Taking into account the size of the pores and their spacing, the mesoporous layer may be though of as a continuous distribution of interfaces, each radiating coherently a wave in all directions, as shown by angularly localized interferences in the scattered light as well as in reflectance. Then reflectance analysis, as interpreted in terms of Fresnel relations cannot give reliable results as far as strong scattering remains involved. Another approach is necessary.

The determination of the effective refractive index in strongly scattering media is studied in a recent article [27] where the scattered light transmitted through a porous GaP sample does not show interferences. The analysis shows that the transport of light is well described by the diffusion approximation, provided it takes place in a non-localized regime that is without interferences. In our case, interferences in the angular distribution of the reflected scattered light have been put in evidence. However, due to the structure of our sample, reflectance and transmittance should exhibit complementary interferences, if any. If we assume that the scattering mean-free path $l_{\mathrm{s}}$ is the order of the distance between pores, then $2 \pi l_{\mathrm{S}} / \lambda<<1$; as a consequence, Anderson localization may take place and interferences should occur. It is then reasonable to conclude that Anderson localization occurs in our system and we can understand a posteriori why any tentative interpretation with simple Fresnel analysis fails.

\section{Conclusion}

In this paper, we have studied the reflection of a Si substrate covered by layers of silica with a mesoporous structure. Interferences, in both reflectance and scatterd light, are the main characteristics of our samples. It has been shown that such specific optical properties should not be interpreted with Fresnel relations without great care. The physical meaning of values of indices has been examined, the Kramers-Kronig relations showing that numerical solutions may be misleading. Arguments have been presented that favor an interpretation of reflectance taking into account the possibility of Anderson localization, as suggested by interferences in the angular distribution of the scattered light. In all cases, these studies will enhance the insight in the peculiar and promising optical properties of mesoporous films.

\section{References}

[1] J.S. Beck, J.C. Vartuli, W.J. Roth, M.E. Leonowicz, C.T. Kresge, K.D. Schmitt, C.T.W. Chu, D.H. Olson, E.W. Sheppard, S.B. McGullen, J.B. Higgins, J.L. Schlenk, J. Am. Chem. Soc. 114 (1992) 10834.

[2] Y. Lu, R. Ganguli, C.A. Drewien, M.T. Anderson, C.J. Brinker, W. Gong, Y. Guo, H. Soyez, B. Dunn, M.H. Huang, J.I. Zink, Nature 389 (1997) 364.

[3] M. Klotz, A. Ayral, C. Guizard, L. Cot, J. Mater. Chem. 10 (2000) 663.

[4] M. Klotz, P.-A. Albouy, A. Ayral, C. Ménager, D. Grosso, A. Van der Lee, V. Cabuil, F. Babonneau, C. Guizard, Chem. Mater. 12 (2000) 1721.

[5] H. Fan, H.R. Bentley, K.R. Kathan, P. Clem, Y. Lu, C.J. Brinker, J. NonCryst. Solids 285 (2001) 79.

[6] C.-M. Yang, A.-T. Cho, F.-M. Pan, T.-G. Tsai, K.-J. Chao, Adv. Mater. 13 (2001) 1099.

[7] F.K. de Theije, A.R. Balkenende, M.A. Verheijen, M.R. Baklanov, K.P. Mogilnikov, Y. Furukawa, J. Phys. Chem., B 107 (2003) 4280.

[8] R.C. Hayward, P. Alberius-Henning, B.F. Chmelka, G.D. Stucky, Microporous Mesoporous Mater. 44-45 (2001) 619.

[9] G. Wirnsberger, B.J. Scott, G.D. Stucky, Chem. Commun. (2001) 119.

[10] G. Wirnsberger, P. Yang, B.J. Scott, B.F. Chmelka, G.D. Stucky, Spectrochim. Acta, Part A: Mol. Biomol. Spectrosc. 57 (2001) 2049.

[11] B.J. Scott, G. Wirnsberger, G.D. Stucky, Chem. Mater. 13 (10) (2001) 3140 .

[12] J. Wang, G.D. Stucky, Advanced Functional Materials, Mesostructured Composite Materials for Multibit-per-Site Optical Data Storage, 2004 $\left(14 \mathrm{n}^{\circ} 5\right)$.

[13] P. Cheyssac, M. Klotz, E. Søndergård, V.A. Sterligov: "Scattering properties of ordered mesoporous silica films" accepted for publication, Optics Communications 2005.

[14] S. Besson, T. Gacoin, C. Jacquiod, C. Ricolleau, D. Babonneau, J.-P. Boilot, Structural study of 3D-hexagonal mesoporous spin-coated sol-gel films, J. Mater. Chem. 10 (2000) 1331.

[15] S. Besson, C. Ricolleau, T. Gacoin, C. Jacquiod, J.-P. Boilot, J. Phys. Chem., B 1043 (2000) 12095.

[16] D. Grosso, F. Cagnol, J. Galo, A.A. de Soler-Illia, E.L. Crepaldi, H. Amenitsch, A. Brunet-Bruneau, A. Bourgeois, C. Sanchez, Adv. Funct. Mater. 14 (4) (2004) 309.

[17] D. Grosso, R. Balkenende, P.A. Albouy, A. Ayral, H. Amenitsch, F. Babonneau, Chem. Mater. 13 (2001) 1848.

[18] M. Klotz, S. Besson, C. Ricolleau, F. Bosc, A. Ayral, Mater. Res. Soc. Symp. Proc. 752 (2003) AA8.6.1.

[19] S. Besson, T. Gacoin, C. Ricolleau, C. Jacquiod, J.-P. Boilot, Nano Lett. 2 (2002) 409.

[20] V.A. Sterligov, P. Cheyssac, "Appareil et procédé de caractérisation optique d'un objet," CNRS patent $n^{\circ} 0115232,23.11 .2001$.

[21] R. Kofman, P. Cheyssac, J. Richard, Phys. Rev., B 16 (1977) 5216.

[22] Virginia Semiconductor, Inc. 1501 Powhatan Street, Fredericksburg, Va 22401 "Optical Properties of Silicon" www.vriginiasemi.com.

[23] C.M. Herzinger, B. Johs, W.A. McGahan, J.A. Woollam, W. Paulson, J. Appl. Phys. 83 (6 15) (1998) 3323.

[24] V. Sterligov, private communication, in press in Appl. Opt.

[25] J. Müllerová, J. Mudro, Acta Phys. Slovaca 50 (2000) 477.

[26] J.N. Hodgson, Optical Absorption and Dispersion in Solids, Chapman and Hall, London, 1970, chapter 1.

[27] J. Gomez Rivas, D.H. Dau, A. Imhof, R. Sprika, B.P.J. Bret, P.M. Johnson, T.W. Hijmans, A. Lagendijk, Opt. Commun. 220 (2003) 17. 